\title{
Diretrizes de Acessibilidade para Jogos Sérios Destinados aos Aprendizes no Espectro Autista
}

\author{
Wendell Soares Pereira ${ }^{1}$, Gilberto Amado de Azevedo Cysneiros Filho ${ }^{1}$, Yuska \\ Paola Costa Aguiar ${ }^{2}$
}
${ }^{1}$ Programa de Pós-Graduação em Informática Aplicada (PPGIA) - Universidade Federal Rural de Pernambuco (UFRPE) - Recife - Pernambuco - Brasil
${ }^{2}$ Departamento de Ciências Exatas (DCX) - Universidade Federal da Paraíba (UFPB) Rio Tinto - Paraíba - Brasil
\{Wendell.pereira, yuska\}@dcx.ufpb.br, g.cysneiros@gmail.com

\begin{abstract}
Serious games have learning as their central goal. They can be an important support tool in the process of acquiring or training skills by people in the Autism Spectrum Disorder (ASD). However, developing serious games for these users can prove challenging. Therefore, this work proposes a set of 109 accessibility guidelines to guide the development of serious games for users with ASD. The set of guidelines was obtained from the analysis of the literature, from a mapping between recommendations for the development of serious games and those aimed at the development of software solutions for users with $A S D$.
\end{abstract}

Resumo. Jogos sérios têm como objetivo central a aprendizagem. Estes podem ser um importante instrumento de apoio ao processo de aquisição ou treinamento de habilidades por pessoas no TRasntorno do Espectro Autista (TEA). No entanto, o desenvolvimento de jogos sérios para estes usuários pode mostrar-se desafiador. Portanto, este trabalho propõe um conjunto de 109 diretrizes de acessibilidade para guiar o desenvolvimento de jogos sérios para usuários com TEA. O conjunto de diretrizes foi obtido a partir da análise da literatura, através de um mapeamento entre recomendações para o desenvolvimento de jogos sérios e àquelas destinadas ao desenvolvimento de soluções de software para usuários com TEA.

\section{Introdução}

O desenvolvimento de diversos setores da sociedade é influenciado pelo uso da tecnologia [Costa et al. 2003]. Dentre estes, tem-se a adoção da tecnologia como estratégia para promoção de integração e inclusão social das Pessoas com Deficiência (PcD). Quando os recursos de tecnologia são destinados especificamente para este público, são intitulados de Tecnologia Assistiva (TA). O Comitê de Ajudas Técnicas [CAT 2007] define a Tecnologia Assistiva como uma área de conhecimento interdisciplinar, que engloba recursos, metodologias, estratégias, práticas e serviços que objetivam promover a funcionalidade relacionada à atividade e participação de Pessoas com Deficiência (permanente ou temporária), incapacidades ou mobilidade reduzida, visando sua autonomia, independência, qualidade de vida e inclusão social.

O número de indivíduos com deficiência no Brasil é de aproximadamente 24,5 milhões, equivalente à $14.5 \%$ do total da população [Censo 2010]. De maneira mais 
específica, estima-se que existam 1,5 milhões de brasileiros com Transtorno do Espectro Autista (TEA), com significativo crescimento no número de casos diagnosticados [Mello et al. 2013; Rios et al. 2015]. O TEA está classificado entre os Transtornos do Neurodesenvolvimento, onde estão inclusos o transtorno autista, o transtorno de Asperger e o transtorno global do desenvolvimento sem outra especificação [DSM-5 2014].

$\mathrm{O}$ espectro indica a pluralidade do transtorno no que diz respeito à diversidade de sintomas manifestados nos indivíduos diagnosticados, a saber: (i) Déficit na comunicação e interação social (ex.: déficit na reciprocidade socioemocional, nos comportamentos comunicativos não verbais, na capacidade de desenvolver, manter e compreender relacionamentos); (ii) Padrões restritos e repetitivos de comportamentos, interesses ou atividades (p. ex., movimentos motores, uso de objetos, ou fala repetitiva; interesses fixos; hipo ou hiper-reatividade a estímulos sensoriais) [DSM-5 2014].

Nesse sentido, a fim de responder às necessidades do público diagnosticado TEA, pesquisadores na área da computação desenvolvem soluções baseadas no computador [Avila et al. 2012], uma vez que, segundo Hourcade et al. (2012), o uso de computadores, mais especificamente, aplicações em tablets-multitouch, pode ser um importante aliado no processo de desenvolvimento de habilidades sociais de pessoas com TEA.

Entre as soluções tecnológicas desenvolvidas para usuários presentes no TEA destacadas por Ferreira (2018), estão os jogos sérios, representando 18\% das 33 soluções abordadas no estudo. Jogos sérios são todos os jogos que têm como objetivo central a aprendizagem, independente do contexto de aplicação (educação, treinamento, saúde, etc.), não sendo o seu propósito primário apenas a diversão [Chen and Michael 2005; Whyte et al. 2015; Dörner et al. 2016].

No entanto, encontrar a sinergia entre a diversão e a pedagogia nos jogos educacionais tem se mostrado uma tarefa difícil [Savi and Ulbricht 2008]. E esse desafio torna-se ainda maior quando os jogos sérios são destinados ao público com particularidades sensoriais, cognitivas e/ou motoras. Além de divertir, estes recursos devem buscar exercitar determinadas funções cognitivas do usuário, demandando uma preocupação maior no seu desenvolvimento [Junqueira et al. 2015]. Desse modo, um jogo sério destinado a usuários com tais particularidades precisa apresentar uma boa correlação entre aspectos de jogabilidade, os objetivos de aprendizagem e as necessidades específicas do público com deficiência.

Diante disso, este trabalho apresenta o processo de concepção de um conjunto de 109 diretrizes de acessibilidade para jogos sérios cujos usuários são aprendizes no espectro autista, organizadas em 15 categorias. Para tal, foram analisados um total de 21 artigos contemplando 44 recomendações para o desenolvimento de jogos sérios. Para recursos digitais destinados ao público com autismo, 15 artigos e 81 recomendações foram analisadas. As 109 diretrizes de acessibilidade para jogos sérios cujos usuários são aprendizes no espectro autista foi resultado do mapeamento entre esses dois conjuntos de recomendações. Cada diretriz foi devidamente classificada segundo seu objetivo, podendo estas apresentarem aspectos referentes às características do público com TEA, aspectos de usabilidade, de jogabilidade, de aprendizagem, ou, ainda, aspectos intrísecos ao próprio processo do desenvolvimento de software. 


\section{Transtorno do Espectro Autista}

O Transtorno do Espectro Autista (TEA), como parte dos Transtornos Globais de Desenvolvimento (TGD), caracteriza uma síndrome responsável por diversas manifestações nos indivíduos diagnosticados, como por exemplo, no que se refere às habilidades de comunicação, interação social e comportamental [DSM-5 2014]. A pluralidade de outros transtornos, com suas mais variadas manifestações nos indivíduos diagnosticados, evidencia a caraterística espectral do TEA. Nesse espectro, estão inclusos a síndrome de Asperger e o autismo, por exemplo.

\subsection{Repertório notavelmente restrito de atividades e interesses}

Indivíduos diagnosticados com TEA apresentam padrões restritos e repetitivos de comportamento, interesses ou atividades, apresentando interesse por brincadeiras repetitivas e monótonas [Brito and Vasconcelos 2016]. As capacidades adaptativas geralmente estão abaixo da medida do QI desses usuários e, quando chegam na fase adulta, estes indivíduos podem ter sérias dificuldades em estabelecer sua independência devido a rejeição contínua a "novidades" e à rigidez de sua rotina [DSM-5 2014].

\subsection{Comprometimento das Habilidades de Comunicação}

As dificuldades de comunicação, ocorrendo de maneira não-padronizada, comprometem o indivíduo com TEA em sua capacidade de compartilhar informações, afetando sobretudo a sua capacidade de iniciar ou manter uma conversação. Como característica das pessoas com TEA, o retardo do desenvolvimento da fala é um fator a ser observado. Geralmente, a fala não é desenvolvida no período habitual, no entanto, em casos de autismo de alto funcionamento, a fala se manifesta por meio de enunciados estruturados gramaticalmente. No entanto, a grande maioria dos indivíduos com TEA enfrentam problemas de compreensão da comunicação inicialmente [DSM-5 2014; Brito and Vasconcelos 2016].

\subsection{Comprometimento das Habilidades de Interação Social}

Uma característica presente nos indivíduos com TEA é a dificuldade de interação recíproca, o déficit na reciprocidade socioemocial. Isso se dá pela dificuldade de interpretação do tom de voz e expressões faciais das pessoas ao seu redor, assim como a dificuldade em entender as pessoas como agentes intencionais [Baldassari et al. 2018].

A habilidade de processar, de maneira implícita, uma emoção apresentada compõe um dos fatores de grande importância para a interação social, ou seja, a capacidade de compreender as expressões faciais dos demais indivíduos, para pessoas com autismo, é fator decisivo na sua capacidade de se envolver em um ambiente social.

\section{Jogos Sérios para Usuários com TEA}

Jogos digitais têm sido um importante ambiente para simular situações, treinar determinadas habilidades, onde as crianças não sentem medo de errar ou tomar uma decisão equivocada ao colocar em prática conceitos que serão necessários em um contexto formal [Baldassari et al. 2018]. O uso de recursos digitais por pessoas diagnosticadas com autismo, quando estes são desenvolvidos de maneira a respeitar as limitações físicas e cognitivas destes usuários, pode ser útil, proporcionando um aprendizado preciso e independente [Ploog et al. 2013]. 
Disponibilizar para usuários com autismo qualquer recurso tecnológico, que não tenha sido projetado respeitando suas características específicas, pode ser um risco no que se refere à aceitação do produto por parte dos pais e dos próprios usuários. Por exemplo, no relatório de Bastien et al. (2018), contendo depoimentos de pais, profissionais e crianças com autismo, ao serem questionados sobre os softwares inseridos nas atividades do dia-a-dia das crianças com TEA, entre os pais respondentes houveram alguns que criticaram tais softwares como uma forma de impedimento na interação social destes usuários (devido à interação ser usuário-dispositivo). No entanto, ao acessar a integralidade dos depoimentos dos pais, é possível perceber que os softwares em questão não foram exclusivamente projetados para o público TEA. Ploog et al. (2013) ressalta a importância de manter a integração entre o recurso digital e as instruções humanas (cuidador, professor, pais), esta integração se mostra superior ao uso exclusivo de recursos tecnológicos, assim como o uso exclusivo das instruções diretas-humanas.

\section{Metodologia}

Um trabalho de pesquisa científica precisa ser sistemático, metódico e crítico, a fim de que se conheça cientificamente os aspectos de determinados assuntos [Prodanov and Freitas 2013]. A fim de atender aos objetivos propostos pelo presente estudo, esta pesquisa seguiu as seguintes atividades metodológicas (Figura 1): (i) Etapa A: Especificação de recomendações para o desenvolvimento de jogos sérios, por meio de um mapeamento sistemático na literatura; seguido da categorização das recomendações segundo suas características; e o agrupamento das recomendações similares ou complementares (ii) Etapa B: busca na literatura por recomendações para 0 desenvolvimento de soluções de software para usuários com TEA; mapeamento entre os dois conjuntos de recomendações, identificando e tratando as diretrizes similares e complementares; classificação das diretrizes segundo o seu foco (TEA, Jogos Sérios, ou TEA \& Jogos Sérios simultaneamente); (iii) Etapa C: Classificação (por ordem de relevância) das diretrizes elencadas por este estudo, realizada com o auxílio de profissionais de diferentes áreas que atuam direta ou indiretamente com o indivíduo com TEA; e, (iv) Etapa D: Documentação da pesquisa em formato de artigo.

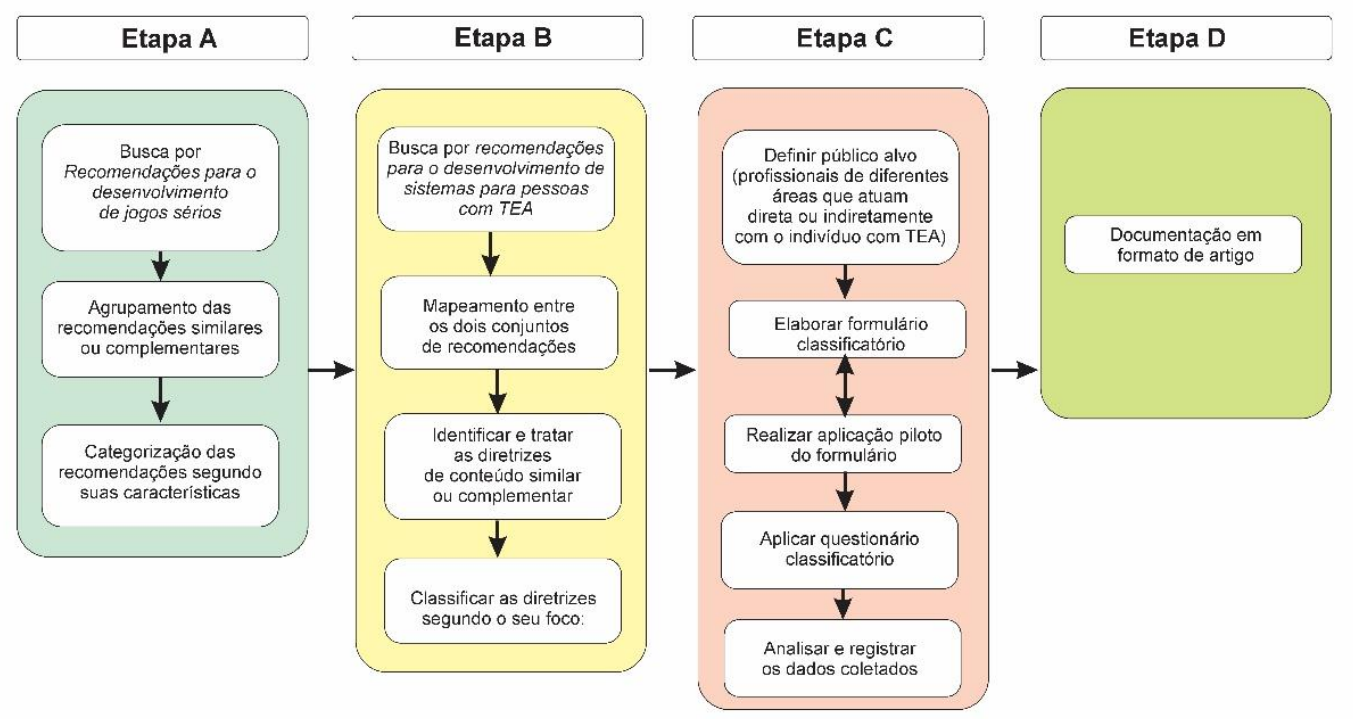

Figura 1. Fluxo de execução das atividades de pesquisa 


\section{Recomendações para o Desenvolvimento de Jogos Sérios}

Diante do objetivo de definir um conjunto de 44 recomendações para o desnvolvimento de jogos sérios, Pereira et al. (2019) realizaram um estudo seguindo as seguintes etapas: (i) Identificação de boas práticas para o desenvolvimento de jogos sérios, a partir de um mapeamento sistemático da literatura; (ii) Agrupamento por similaridade das boas práticas encontradas na literatura; (iii) categorização das diretrizes segundo suas características, podendo serem classificadas como pedagógicas, de usabilidade, de jogabilidade, para softwares em geral, ou ainda, a junção entre essas categorias.

A partir de um corpus formado por 18 artigos, Pereira et al. (2019) identificaram 130 boas práticas para o design e desenvolvimento de jogos sérios que, após um processo de agrupamento (utilizando a similaridade por critério), resultou em um conjunto de 44 recomendações. As recomendações foram categorizadas segundo seu objetivo, podendo uma recomendação conter características de: Usabilidade (12/44); Jogabilidade (14/44); Educacional (5/44); Jogabilidade\&Usabilidade (5/44); Usabilidade\&Educacional (2/44); ou ainda, Recomendações para softwares em geral (6/44), ou seja, intrísecos ao processo de desenvolvimento de software. A Figura 2 destaca alguns exemplos das 44 recomendações encontradas por Pereira et al. (2019).

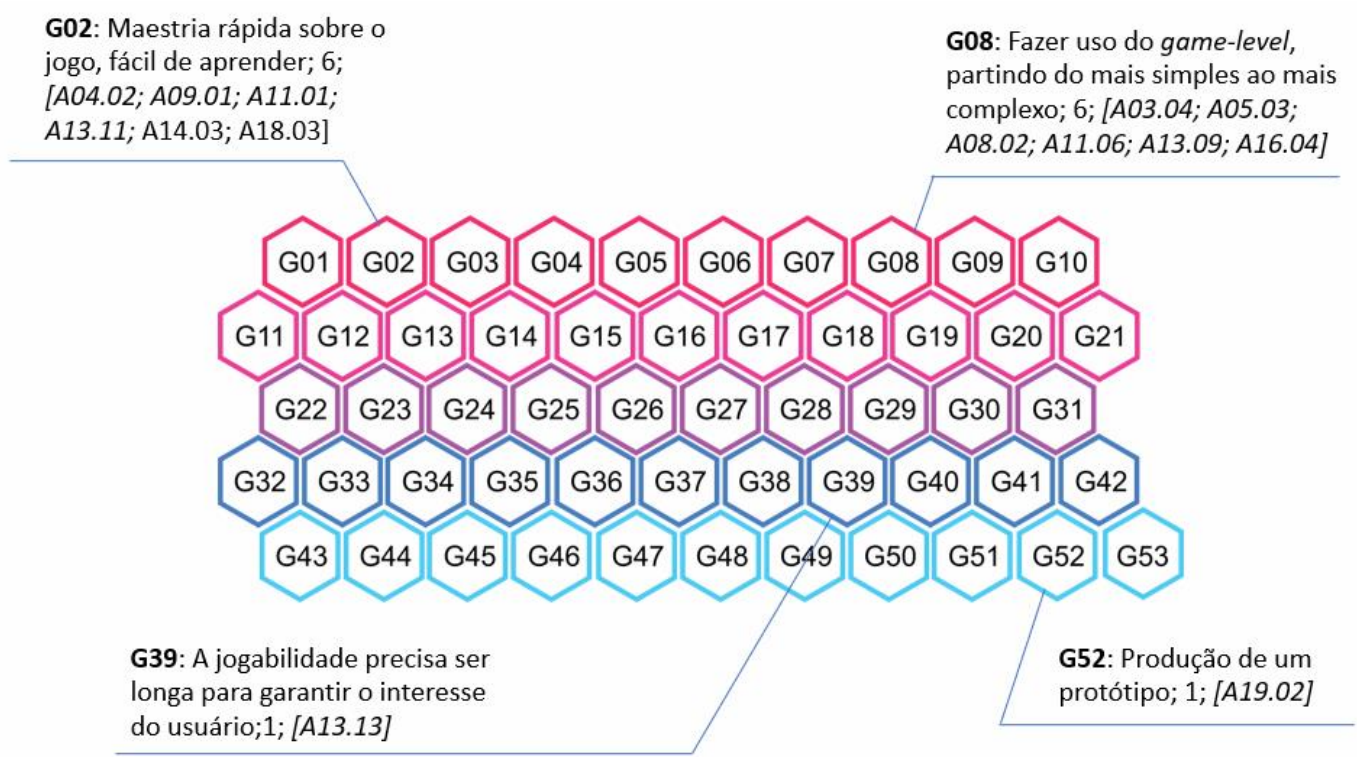

Figura 2. Recomendações para o desenvolvimento de jogos sérios. Fonte: Baseado no artigo de Pereira et al. (2019).

\section{Recomendações para o Desenvolvimento de Soluções de Software para Usuários com TEA}

O desafio de desenvolver soluções de software para usuários com TEA, sendo estes jogos ou não, tem sido sujeito de pesquisa de muitos estudos [Britto and Pizzolato 2018; Magaton and Bim 2019; Aguiar et al. 2020], provenientes de diversas áreas de 
conhecimento. A partir desse desafio, pesquisadores buscam aliar boas práticas de desenvolvimento de software às necessidades específicas dos indivíduos com TEA.

\subsection{GAIA: uma proposta de um guia de recomendações de acessibilidade de interfaces $W e b$ com foco em aspectos do Autismo}

Na pesquisa realizada por Britto e Pizzolato (2018), é apresentado o desenvolvimento do projeto GAIA (Guia de Acessibilidade de Interfaces Web com foco em aspectos do Autismo), que consistiu em um conjunto de recomendações de design para acessibilidade a fim de apoiar os desenvolvedores e educadores no processo de desenvolvimento de websites mais adequados às necessidades de crianças com TEA.

Nessa pesquisa, Britto e Pizzolato (2018) extraíram 107 recomendações, a partir de uma revisão bibliográfica exploratória (considerando o período de 2005-2015) que considerou 17 trabalhos, entre pesquisas científicas e produtos comerciais de softwares voltados a crianças com autismo. Essas recomendações foram normalizadas (recomendações similares que podiam ser conectadas) em 28 recomendações únicas (disponíveis em gaia.wiki.br), organizadas em 10 categorias.

\subsection{Recomendações para o desenvolvimento de softwares voltados para crianças com transtorno do espectro autista}

Com o objetivo de elencar recomendações para o desenvolvimento de softwares voltados para crianças com TEA, Magaton e Bim (2019) combinaram resultados de uma revisão de literatura com os dados obtidos de uma pesquisa de campo (observação da interação).

Das 17 recomendações resultantes da revisão bibliográfica, os autores puderam confimar a importância de apenas 12 delas, durante a observação da interação envolvendo crianças com TEA, seus responsáveis e profissionais especializados em TEA. Além destas, com base na observação e nas informações coletadas com os pais/responsáveis e profissionais de TEA, os autores propuseram ainda outras 7 recomendações.

\subsection{Recomendações para o desenvolvimento de soluções de software para usuários com transtorno do espectro Autista}

Com a finalidade de identificar recomendações que pudessem subsidiar o design e o desenvolvimento de sistemas adequados para usuários com TEA, Aguiar et al. (2020) propuseram uma revisão sistemática que considerou 15 estudos, de onde foram extraídas 155 recomendações.

Após uma análise realizada sobre as 155 recomendações encontradas, notou-se que algumas delas eram similares ou se complementavam. Depois de submeter as 155 recomendações a uma análise avaliando a similaridade, complementariedade e especificidade, foi gerada uma lista contendo 81 recomendações. No entanto, as recomendações não apresentavam uma terminologia comumente utilizada entre os profissionais de IHC, o que poderia dificultar o uso desse instrumento por parte dos profissionais da área.

As 81 recomendações foram organizadas de acordo com os Critérios Ergonômicos (adaptabilidade, orientação, carga de trabalho, compatibilidade, etc.) propostos por Bastien \& Scapin (1993). Após uma análise realizada pelos especialistas, algumas recomendações não foram combinadas com os Critérios Ergonômicos. Por isso, além do 
que foi proposto por Bastien \& Scapin (1993), foram criados três outros grupos para classificar as recomendações: (i) Princípios gerais de usabilidade; (ii) Requisitos funcionais; (iii) Requisitos não-funcionais. Na Figura 3, são exibidos alguns exemplos das 81 recomendações especificadas por Aguiar et al. (2020) no AutismGuide.

Carga de Trabalho

Compatibilidade

Orientação

WKLD_04: Evitar a grande quantidade simultânea de recursos, imagens, animações, etc.;

CMPT_01: Considerar os aspectos demográficos dos usuários (idade, sexo, etc.);

GUID_13: Apresentar informações complementares à tarefa (objetivos, duração esperada, número de etapas, ...);

Figura 3. Exemplo de recomendações para o de desenvolvimento de soluções de software para usuários com TEA

\subsection{Análise comparativa entre as propostas abordadas}

As propostas apresentadas nesta seção, de maneira comum, têm por objetivo direcionar o desenvolvimento de soluções tecnológicas para o público com TEA. De maneira similar, Britto e Pizzolato (2018), Magaton e Bim (2019) e Aguiar et al. (2020) apresentam recomendações que combinam aspectos de interface e interação às particularidades apresentadas pelo público com TEA. No entanto, aspectos pedagógicos e de jogabilidade não são explicitamente tratados. Por esse motivo, buscou-se no presente trabalho unir o conjunto recomendações para o desenvolvimento de jogos sérios aos aspectos necessários para o desenvolvimento de soluções tecnológicas para usuários com TEA.

Na Figura 3, é apresentada uma análise comparativa entre os três conjuntos de recomendações apresentados nesta seção. A partir dessa tabela, nota-se o maior número de recomendações no trabalho proposto por Aguiar et al. (2020), apesar de não ter abordado o maior número de estudos na revisão sistemática. Entre o conjunto de 81 recomendações proposto por Aguiar et al. (2020) incluem as recomendações de Britto e Pizzolato (2018), apresentando igualmente recomendações similares às propostas por Magaton e Bim (2019). Por esse motivo, o conjunto de diretrizes proposto por Aguiar et al. (2020) mostra-se o mais abrangente que os demais, sendo escolhido para compor a combinação com o conjunto de recomendações para o desenvolvimento de jogos sérios.

\begin{tabular}{|l|c|c|c|c|c|c|}
\hline \multicolumn{8}{|c|}{ Comparativo Entre os Conjuntos de Recomendações Apresentados } \\
\hline & \multicolumn{3}{|c|}{ Aspectos abordados em cada proposta } & Quantitativo de cada proposta \\
\hline & Usabilidade & Jogabilidade & $\begin{array}{c}\text { Especificos } \\
\text { para TEA }\end{array}$ & Pedagógicos & $\begin{array}{c}\text { Total de } \\
\text { Estudos } \\
\text { Considerados }\end{array}$ & $\begin{array}{c}\text { Total de } \\
\text { Recomendações }\end{array}$ \\
\hline Britto e Pizzolato (2018) & Sim & Não & Sim & Não & 17 & 28 \\
\hline Magaton e Bim (2019) & Sim & Não & Sim & Não & 5 & 17 \\
\hline Aguiar et al. (2020) & Sim & Não & Sim & Não & 15 & 81 \\
\hline
\end{tabular}

Figura 4. Comparativo entre os conjuntos de recomendações para o desenvolvimento de soluções de software para usuários com TEA. Fonte: 0 próprio autor 


\section{Diretrizes para o Desenvolvimento de Jogos Sérios para Usuários no Espectro Autista}

O processo de obtenção da lista de diretrizes para o desenvolvimento de jogos sérios para pessoas com autismo foi composto pela combinação de duas outras listas de recomendações: (i) recomendações para o desenvolvimento de jogos sérios em geral Pereira et al. (2019); e (ii) recomendações para o desenvolvimento de sistemas para pessoas com TEA [Aguiar et al. 2020]. Desse modo, como resultado desse processo de combinação de recomendações, tem-se uma lista que contempla diretrizes, representando os elementos necessários para o desenvolvimento de jogos sérios para usuários com TEA, contemplando os aspectos pedagógicos, de engenharia de software e de jogabilidade. Dessa maneira, as diretrizes podem servir como um instrumento de auxílio no desafio que é desenvolver jogos sérios para usuários com TEA, buscando manter a sinergia entre a diversão, a aprendizagem e as necessidades específicas do público com TEA.

\subsection{Mapeamento entre as diretrizes para o desenvolvimento de sistemas para usuários com TEA e as diretrizes de desenvolvimento de jogos sérios}

Nesta etapa, o autor deste trabalho (pesquisador da área de IHC), sob a supervisão de seus orientadores (pesquisadores na área de IHC e Engenharia de Software), identificou as similaridades entre os dois conjuntos de recomendações, a fim de eliminar as duplicidades e evitar redundâncias. $\mathrm{O}$ mapeamento entre os dois conjuntos de diretrizes foi registrado

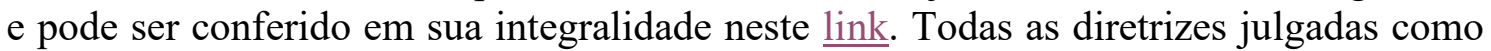
similares sofreram alterações no seu texto descritivo, uma vez que a diretriz atualizada precisava contemplar informações presentes na versão original de ambas.

Durante esse processo foram notificadas 16 similaridades identificadas nas seguintes categorias: requisitos funcionais (3/16); requisitos não funcionais (1/16); carga de trabalho (1/16); consistência (1/16); gerenciamento de erros (1/16); compatibilidade (1/16); controle explícito do usuário (1/16); adaptabilidade (3/16); e, orientação (4/16). Importante destacar que nenhuma diretriz para jogos sérios possuiu características similares à nenhuma recomendação presente na categoria de significado dos códigos, fazendo desta a única categoria não contemplada pelas diretrizes para o desenvolvimento de jogos sérios.

\subsection{Diretrizes para o desenvolvimento de jogos sérios para usuários no espectro Autista}

Unindo os dois conjuntos de recomendações, considerando todas as similaridades existentes, um total de 109 diretrizes para o desenvolvimento de jogos sérios para usuários com TEA. Todas as diretrizes foram organizadas segundo a sua categoria, podendo estas apresentarem características das heurísticas de usabilidade (requisitos funcionais, requisitos não-funcionais, carga de trabalho, significado dos códigos, consistência, gerenciamento de erros, compatibilidade, controle explícito, adaptabilidade e orientação), características pedagógicas, de jogabilidade ou diretrizes gerais sobre o desenvolvimento do jogo sério. Além disso, foi observado o foco que cada diretriz possuía, podendo variar segundo seus objetivos. As diretrizes puderam ainda ser categorizadas segundo seu foco. Desse modo, 27 diretrizes tiveram seu foco em jogos sérios (SG), 65 são específicas para Transtorno do Espectro Autista (TEA), enquanto 16 focam nos dois aspectos simultaneamente (TEA\&SG). 
Todas as 109 diretrizes foram organizadas de acordo com as categorias propostas por Aguiar et al. (2020), onde cada categoria representa uma heurística de usabilidade proposta por Bastien e Scapin (1993). Em adição a essas categorias, foram criadas as categorias para os aspectos pedagógicos, de jogabilidade, de desenvolvimento e para aspectos transversais.

Importante ressaltar que o conjunto de diretrizes apresentado por esta pesquisa é um instrumento de auxílio para o processo de desenvolvimento, mas que não substitui aspectos esseciais como uma equipe multidisciplinar e/ou o design centrado no usuário, por exemplo. Na Figura 5, são apresentados alguns exemplos de diretrizes presentes no conjunto de 109, organizadas em 15 categorias, subdivididas de acordo com seu foco: jogos sérios (azul), TEA (vermelho) e jogos sérios \& TEA (roxo). Cada diretriz contém seu identificador único (ID), a descrição da diretriz em si e, por fim, as referências utilizadas para a construção dessa diretriz. O conjunto das 109 diretrizes pode ser encontrado de maneira completa, disponível online.

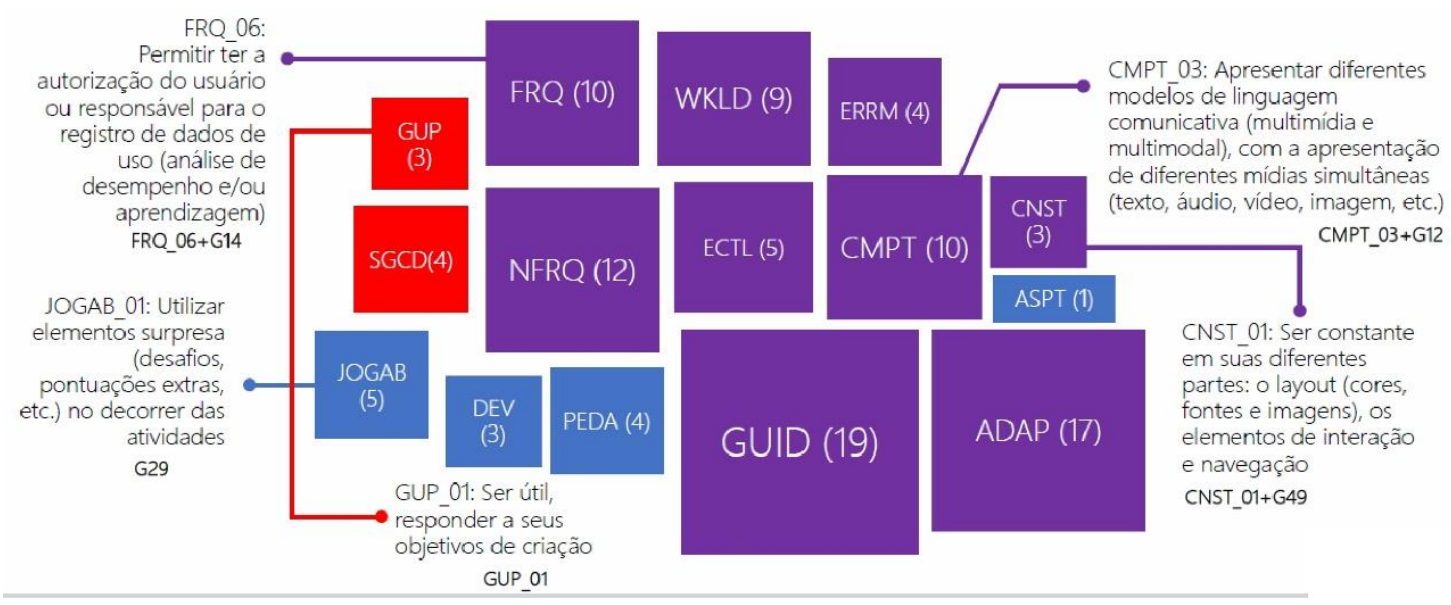

\section{Figura 5. Recomendações para o desenvolvimento de jogos sérios para usuários com TEA}

\subsection{Classificação das Diretrizes por Ordem de Prioridade}

Como tentativa de classificar, por ordem de prioridade, as diretrizes elencadas, foi elaborado um formulário online, onde profissionais/pesquisadores/cuidadores (que possuíam contato com indivíduos diagnosticados com TEA) foram convidados a ranquear as diretrizes subdivididas por categorias. No entanto, apesar da divulgação realizada, o número de respondentes foi insuficiente para qualquer análise estatística. Por esse motivo, a etapa do ranqueamento das diretrizes, como parte integrante desta pesquisa, não pôde ser completada.

\section{Considerações Finais}

A presente pesquisa se propôs a identificar um conjunto de 109 diretrizes para apoiar o processo de desenvolvimento de jogos sérios para usuários com TEA. Para isso, foi realizado um mapeamento entre um conjunto de recomendações para o desenvolvimento de jogos sérios e um conjunto de recomendações para o desenvolvimento de soluções de software para usuários com TEA, ambos encontrados na literatura. Durante esse processo, as similaridades e complementaridades foram identificadas e devidamente tratadas. As 
diretrizes foram organizadas de acordo com as categorias especificadas no estudo de Aguiar et al. (2020). Por fim, as diretrizes ainda puderam ser categorizadas segundo seu objetivo de aplicação, onde cada diretriz poderia ter seu foco em jogos sérios, em TEA ou nos dois simultaneamente (jogos sérios+TEA).

No entanto, as diretrizes desenvolvidas por esta pesquisa não foram devidamente classificadas por profissionais/cuidadores que lidam diretamente com o público com TEA. Esta etapa da pesquisa foi desenvolvida durante a crise pandêmica do COVID-19, o que pode ter impedido a ampla divulgação do formulário classificatório. Além disso, a verificação da aplicabilidade das diretrizes elencadas neste trabalho não estava no escopo incial do estudo. Porém, sabe-se da relevância de verificar a aplicabilidade do conjunto de diretrizes no processo de desenvolvimento de um jogo sério para usuários com TEA. Por esse motivo, como etapa futura dessa pesquisa sugere-se o desenvolvimento de um jogo sério para usuários com TEA, considerando o conjunto de diretrizes resultante desta pesquisa. E, com base nos dados fornecidos pela equipe de desenvolvimento, verificar a aplicabilidade das diretrizes no contexto de desenvolvimento de software.

\section{Agradecimentos}

À Coordenação de Aperfeiçoamento de Pessoal de Nível Superior - Brasil (CAPES) Código de Financiamento 001, pelo financiamento desse projeto de pesquisa. Ao Conselho Nacional de Desenvolvimento Científico e Tecnológico (CNPq), pelo financiamento via projeto Universal (MCTIC/CNPq No28/2018,425551/2018-0).

\section{Referências}

Boulic, R. and Renault, O. (1991) "3D Hierarchies for Animation”, In: New Trends in Animation and Visualization, Edited by Nadia Magnenat-Thalmann and Daniel Thalmann, John Wiley \& Sons ltd., England.

Aguiar, Y. P. C., Galy, E., Godde, A., Trémaud, M. and Tardif, C. (2020). AutismGuide: a usability guidelines to design software solutions for users with autism spectrum disorder. Behaviour \& Information Technology, 1-19.

Avila, B. G., Passerino, L. M. and Reatequi, E. (2012). Proposta de uma metodologia para a construção de um sistema de CAA focado no contexto de seus usuários. Revista Brasileira de Informática na Educação, 20(1), 97.

Baldassarri, S., Passerino, L., Ramis, S., Riquelme, I. and Perales, F. J. (2018, September). Videogame-based case studies for improving communication and attention in children with ASD. In Proceedings of the XIX International Conference on Human Computer Interaction (pp. 1-8).

Bastien, C. and Scapin, D. L. (1993). Ergonomic criteria for the evaluation of humancomputer interfaces (Doctoral dissertation, Inria).

Bastien, R., Garnier, P., Haelewyck, M. C., Judge, H., Martin, J. C., Sellers, T. and Thommen, E. (2018). Autisme et nouvelles technologies: témoignages des parents, professionnels et enfants autistes sur les usages numériques. Rapport inter-pays (Doctoral dissertation, FIRAH; INSHEA-Institut national supérieur de formation et de recherche pour l'éducation des jeunes handicapés et les enseignements adaptés). 
Brito, A. R. and Vasconcelos, M. M. D. (2016). Conversando sobre autismoreconhecimento precoce e possibilidades terapêuticas. CAMINHA, Vera Lúcia; HUGUERIN, Juliane; ASSIS, Lúcia M.: e ALVES, Priscila Pires. Autismo: vivências e Caminhos. São Paulo: Blucher, 3.

Britto, T. C. P. and Pizzolato, E. B. (2018). GAIA: uma proposta de um guia de recomendações de acessibilidade de interfaces Web com foco em aspectos do Autismo. Revista Brasileira de Informática na Educação, 26(02), 102.

CAT, C. d. A. T. (2007). Ata da reunião VII, de dezembro de 2007 do comitê de ajudas técnicas. Secretaria Especial dos Direitos Humanos da Presidência da República (CORDE/SEDH/PR).

CENSO, I. (2010). Disponível em: < http://www. censo2010. ibge. gov. br/>. Acesso em, v. 23.

Chen, S. and Michael, D. (2005). Proof of learning: Assessment in serious games. Retrieved October, 17, 2008.

Costa, V. M., Rapkiewicz, C. E., de Queirós Filho, M. G. and Canela, M. C. (2003). Avaliação de sites educacionais de Química e Física: um estudo comparativo. In Anais do Workshop de Informática na Escola (Vol. 1, No. 1, pp. 545-554).

Dörner, R., Göbel, S., Effelsberg, W. and Wiemeyer, J. (2016). Serious games. Basel, Switzerland: Springer International Publishing.

DSM-5, A. (2014). DSM-5: Manual diagnóstico e estatístico de transtornos mentais. [S.1.]: Artmed Editora.

Ferreira, W., Cordeiro, R., Aguiar, Y. P. C., Saraiva, J., Tardif, C., and Galy, E. (2018, October). Panorama das Publicações Nacionais sobre Autismo, Educação e Tecnologia. In Brazilian Symposium on Computers in Education (Simpósio Brasileiro de Informática na Educação-SBIE) (Vol. 29, No. 1, p. 913).

Hourcade, J. P., Bullock-Rest, N. E. and Hansen, T. E. (2012). Multitouch tablet applications and activities to enhance the social skills of children with autism spectrum disorders. Personal and ubiquitous computing, 16(2), 157-168.

Junqueira, M. A., Cunha, L. F., Ribeiro, J. G. and Machado, A. (2015, October). Uma proposta de jogo assistivo para dispositivos móveis em prol da inclusão digital de deficientes visuais. In Anais do Workshop de Informática na Escola (Vol. 21, No. 1, p. 554).

Magaton, H. C. and Bim, S. A. (2019). Recomendações para o Desenvolvimento de Softwares Voltados para Crianças com Transtorno do Espectro Autista. Revista Brasileira de Informática na Educação, 27(02), 112.

Mello, A. M., Andrade, M. A. and Chen, H. H. (2013). Retratos do autismo no Brasil. São Paulo: Associação dos Amigos do Autista.".

Michael, D. R. and Chen, S. L. (2005). Serious games: Games that educate, train, and inform. Muska \& Lipman/Premier-Trade.

Pereira, W. S., Cysneiros, G., \& Aguiar, Y. P. C. (2019). Diretrizes para o Desenvolvimento de Serious Games: Um Mapeamento Sistemático da Literatura. In 
Brazilian Symposium on Computers in Education (Simpósio Brasileiro de Informática na Educação-SBIE) (Vol. 30, No. 1, p. 714).

Ploog, B. O., Scharf, A., Nelson, D. and Brooks, P. J. (2013). Use of computer-assisted technologies (CAT) to enhance social, communicative, and language development in children with autism spectrum disorders. Journal of autism and developmental disorders, 43(2), 301-322.

Prodanov, C. C., \& De Freitas, E. C. (2013). Metodologia do trabalho científico: métodos e técnicas da pesquisa e do trabalho acadêmico-2a Edição. Editora Feevale.

Rios, C., Ortega, F., Zorzanelli, R. and Nascimento, L. F. (2015). From invisibility to epidemic: the narrative construction of autism in the Brazilian press. InterfaceComunicação, Saúde, Educação, 19(53), 325-336.

Savi, R. and Ulbricht, V. R. (2008). Jogos digitais educacionais: benefícios e desafios. Renote, 6(1).

Whyte, E. M., Smyth, J. M. and Scherf, K. S. (2015). Designing serious game interventions for individuals with autism. Journal of autism and developmental disorders, 45(12), 3820-3831. 